\title{
Operating partial core transformers under liquid nitrogen conditions
}

\author{
M.C.Liew, M.B.O'Neill and P.S.Bodger
}

\begin{abstract}
A reverse design method has been applied to partial core transformers. Three sample transformers were built and then tested under liquid nitrogen $\left(\mathrm{N}_{2}\right)$ conditions. The measured results were compared to model calculated values and the differences used to modify the reverse design model in order to more accurately determine the calculated equivalent circuit parameters. A fourth transformer was then designed, built and tested. The measured performance of this transformer confirmed the validity and accuracy of the model, and showed the usefulness of the reverse design approach.
\end{abstract}

\section{Introduction}

Equivalent circuit modelling has been widely used in designing transformers. Recently, a reverse design transformer model has been introduced [1], which derives circuit components from the characteristics and dimensions actually used to build transformers. Physical characteristics and dimensions of the windings and core are the specifications. By manipulating the amount and type of material actually to be used in the construction of the transformer, its performance can be determined. Such an approach lends itself to designing transformers using what is available from suppliers. This is essentially the opposite of the conventional design approach.

This approach offers much flexibility in designing to user performance criteria and, for the transformers studied, has shown improved accuracy in predicting performance as compared to the conventional transformer modelling approach [2]. While transformer manufacturers may well have more accurate modelling techniques, these are likely to be custom designed rather than what is freely available in the public literature.

This reverse design approach has since been applied to partial core transformers [3], where the return yokes and limbs of a full core transformer have been removed. Partial core transformers are being studied because the size of their core can be dramatically reduced albeit by an increase in winding turns. The combination gives better magnetisation than a coreless transformer and maintains the leakage flux at an acceptably low level. The combination also means that the overall weight of the partial core units is significantly reduced, and they are easier to manufacture. The calculated and measured operational performances of these transformers at normal operating temperatures have shown good agreement [3].

Conventional full core transformers have been known to perform differently under different operating temperatures

\footnotetext{
(C) IEE, 2001

IEE Proceedings online no. 20010438

DOI: $10.1049 /$ ip-epa:20010438

Paper first received 27th November 2000 and in revised form 4th April 2001

The authors are with the Department of Electrical and Electronic Engineering, University of Canterbury, Private Bag 4800, Christchurch, New Zealand
}

[4]. The resistivity of metals, in particular copper and aluminium, is temperature dependent. The copper losses in liquid nitrogen filled full core transformers have been found to be approximately $30 \%$ of that of traditional oil filled full core transformers [5]. But, while the overall losses have been decreased, the efficiency of the liquid nitrogen generator is such that the combined transformer/generator efficiency is worse than that for oil insulated transformers. Moreover, the electrical and mechanical breakdown strengths of selected insulation are of the same order, if not better, under liquid nitrogen as compared to oil impregnated insulation [6]. No further specific information in the literature has been found regarding the effect of the temperature change to other parameters.

In this paper, partial core transformers are operated in liquid nitrogen to determine their characteristics at a different operating temperature. This is a necessary stage in the development of a partial core superconducting transformer, which may give rise to an overall transformer/generator efficiency comparable to oil filled transformers.

\section{Initial investigations}

Consideration is given to the partial core transformer as depicted in Fig. 1. A laminated core occupies the central space. Windings are wound around the core, with the

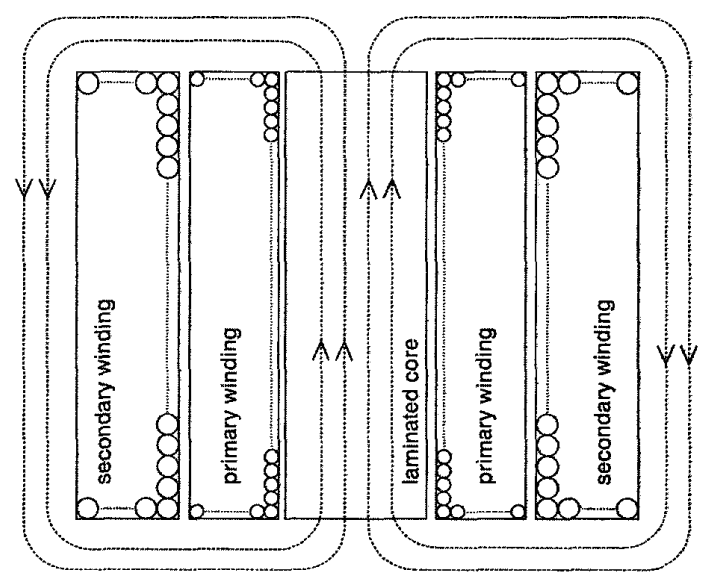

Fig. 1 Cross-sectional view of partial core transformer 
primary winding inside the secondary winding. This was a convenient arrangement for the intended use of the transformers. The yokes and limbs, which usually form the rest of the core in full core transformers, are not present.

The nominal ratings of three partial core transformers for normal operating temperatures and their physical dimensions and material characteristics are listed in Table 1.

Table 1: Transformer design data

\begin{tabular}{llll}
\hline Transformer & no. 1 & no. 2 & no. 3 \\
\hline Ratings & & & \\
$\quad$ Primary voltage, $\mathrm{V}$ & 110 & 230 & 230 \\
$\quad$ Secondary voltage, $\mathrm{V}$ & 28 & 23 & 230 \\
$\quad$ VA rating, kVA & 4.4 & 5.3 & 2.0 \\
$\quad$ Operating frequency, $\mathrm{Hz}$ & 50 & 50 & 50 \\
Core & & & \\
$\quad$ Length, mm & 400 & 140 & 258 \\
$\quad$ Width 1, mm & 31 & 44 & 42 \\
$\quad$ Width 2, mm & 38 & 44 & 43 \\
$\quad$ Lamination thickness, mm & 0.5 & 0.5 & 0.5 \\
$\quad$ Relative permeability & 2000 & 2000 & 2000 \\
Primary winding & & & \\
$\quad$ Number of layers & 3 & 8 & 6 \\
$\quad$ Wire diameter, mm & 2.5 & 2.25 & 2.5 \\
Secondary winding & & & \\
$\quad$ Number of layers & 2 & 2 & 7 \\
$\quad$ Wire diameter, mm & 4.5 & 4.5 & 2.5 \\
\hline
\end{tabular}

Transformers 1 and 2 were arc welders designed for high secondary load current $(\simeq 100 \mathrm{~A})$ applications, each operated at a different supply voltage. Transformer 3 was a prototype 1:1 isolating transformer.

The relative permeabilities of the steel are estimates of open circuit rated values. For design purposes they are considered constant.

To further investigate the precision of the model developed in [3], the performance of the three partial core transformers was measured by immersing them in liquid nitrogen.

Open circuit and short circuit tests were carried out on the transformers. The open circuit tests were carried out at rated primary input voltages, while short circuit tests were carried out at rated input currents. The test details are shown in Table 2.
The open circuit and short circuit tests results, calculated for the transformers using the reverse design method, are also presented in Table 2 for comparison. It can be seen that the reactive components $X_{m}$ and $X_{\text {leak }}$ do not alter significantly. Therefore, changes in the operating temperature of the transformers have a negligible effect on the reactive components. This is an expected result. However, the core loss component $R_{c}$ is notably different between the calculated and measured values, thus a better calculation is required to account for low temperature operation. Moreover, while it can been seen from Table 2 that the calculated and measured values of the combined winding resistance $R_{\text {wind }}$ appear to be in close agreement, this is misleading. The resistivity of copper wire at low temperatures is different from that calculated using conventional formulae and also the actual wire temperature will be higher than the immersion liquid temperature.

\subsection{Effects of temperature changes on the windings}

$R_{\text {wind }}$ is a combination of the primary and secondary winding resistances $\left(R_{1}\right.$ and $\left.R_{2}\right)$, referred to the primary, This is depicted in the equivalent circuit model in Fig. 2 [7].

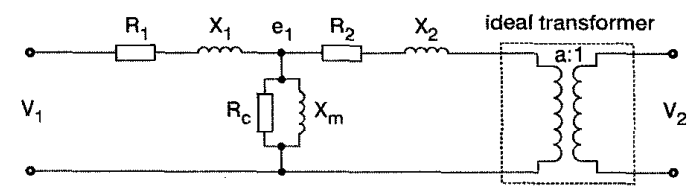

Fig.2 Transformer equivalent circuit, referred to primary winding

$R_{1}$ and $R_{2}$ can be calculated using:

$$
R=\rho \frac{l}{A}
$$

where $\rho=$ resistivity of the conductor

$l=$ length of the conductor

$A=$ cross-sectional area of the conductor

The resistivity of a given conductor varies with its temperature. Consequently, by immersing the transformers into liquid nitrogen $\left(77 \mathrm{~K}\right.$ or $\left.-196^{\circ} \mathrm{C}\right)$, the windings' resistivities drop significantly. In [2], the operating resistivity at temperature $T^{\circ} \mathrm{C}$ is given as:

$$
\rho=\frac{(1+\Delta \rho T) \rho_{20^{\circ} \mathrm{C}}}{(1+20 \Delta \rho)}
$$

where $\Delta \rho=$ thermal resistivity coefficient $\rho_{20^{\circ} \mathrm{C}}=$ material resistivity at $20^{\circ} \mathrm{C}$.

\begin{tabular}{|c|c|c|c|c|c|c|c|c|c|}
\hline \multirow{2}{*}{$\begin{array}{l}\mathrm{o} / \mathrm{c} \text { and } \mathrm{s} / \mathrm{c} \\
\text { test results }\end{array}$} & \multicolumn{2}{|c|}{ Transformer 1} & \multirow[b]{2}{*}{ Error (\%) } & \multicolumn{3}{|c|}{ Transformer 2} & \multicolumn{3}{|c|}{ Transformer 3} \\
\hline & Calc. & Meas. & & Calc. & Meas. & Error $(\%)$ & Calc. & Meas. & Error $(\%)$ \\
\hline$V_{100}, V$ & 110 & 110 & 0 & 230 & 230 & 0 & 230 & 230 & 0 \\
\hline$h_{10 c} \mathrm{~A}$ & 8.1 & 8.4 & 4 & 19.8 & 21.9 & 10 & 10.7 & 10.25 & 4 \\
\hline$P_{10 c}, W$ & 60 & 70 & 14 & 235 & 280 & 16 & 76 & 120 & 37 \\
\hline$p f_{1 o c}$ & 0.07 & 0.08 & 13 & 0.05 & 0.06 & 17 & 0.03 & 0.05 & 40 \\
\hline$R_{C^{\prime}} \Omega$ & 202.4 & 173.1 & 17 & 225.3 & 188.9 & 19 & 695.0 & 440.8 & 58 \\
\hline$X_{m}, \Omega$ & 13.6 & 13.1 & 4 & 11.6 & 10.5 & 10 & 21.4 & 22.5 & 5 \\
\hline$V_{1 s c}, V$ & 11 & 11 & 0 & 40 & 40 & 0 & 12.2 & 12.2 & 0 \\
\hline$I_{1 s c r} \mathrm{~A}$ & 40 & 40 & 0 & 24.1 & 23 & 5 & 8.2 & 8.7 & 6 \\
\hline$P_{1 s c}, \mathrm{~W}$ & 217 & 200 & 8 & 343 & 280 & 22 & 15 & 18 & 17 \\
\hline$p f_{1 s c}$ & 0.49 & 0.44 & 11 & 0.36 & 0.30 & 20 & 0.15 & 0.18 & 17 \\
\hline$R_{\text {wind }} \Omega$ & 0.14 & 0.13 & 8 & 0.59 & 0.53 & 11 & 0.22 & 0.23 & 4 \\
\hline$X_{\text {leak }}, \Omega$ & 0.24 & 0.24 & 0 & 1.55 & 1.66 & 7 & 1.47 & 1.38 & 7 \\
\hline
\end{tabular}

Table 2: Experimental results for the transformers 
For a copper conductor, the resistivity at $20^{\circ} \mathrm{C}$ is $1.76 \times$ $10^{-8} \Omega \mathrm{m}$, and $\Delta \rho=0.0039 \times 10^{-8} \Omega \mathrm{m} /{ }^{\circ} \mathrm{C}$. Using eqn. 2 , the corresponding resistivity at $-196^{\circ} \mathrm{C}$ is calculated to be 3.85 $\times 10^{-9} \Omega \mathrm{m}$.

A plot of the copper resistivity against temperature is found in [8]. A portion of this plot, between $-203^{\circ} \mathrm{C}(70 \mathrm{~K})$ and $127^{\circ} \mathrm{C}(400 \mathrm{~K})$ is reproduced in Fig. 3. The values calculated using eqn. 2 are superimposed on Fig. 3. It can be seen that the resistivity values between the two lines are very close at room temperature $\left(20^{\circ} \mathrm{C}\right)$ and above. Therefore, the use of eqn. 2 for temperatures above $20^{\circ} \mathrm{C}$ is justified. However, there is a significant difference between the two lines at extremely low temperatures. The relative error is as much as $125 \%$. As a result, eqn. 2 needs to be modified to account for the difference at extremely low temperatures.

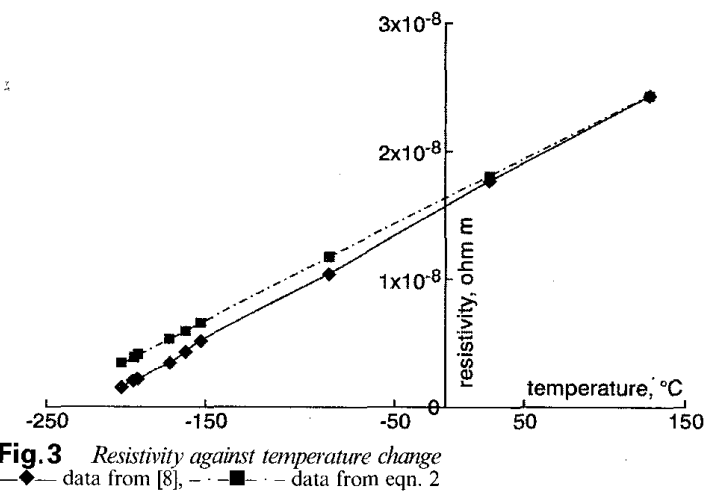

From Fig. 3, it is observed that the line from [8] is almost straight. A linear regression fit of the line is found to give:

$$
\rho=6.99 \times 10^{-11} \mathrm{~T}+1.57 \times 10^{-8}
$$

where $T=$ the operating temperature $\left({ }^{\circ} \mathrm{C}\right)$.

This equation is used to replace eqn. 2 in the model. The relative errors between eqn. 3 and the data line [8] of Fig. 3 are very small. This is shown in Table 3.

Table 3: Comparing resistivity between the data from [8] and eqn. 3

\begin{tabular}{|c|c|c|c|c|}
\hline \multicolumn{2}{|c|}{ Temperature } & \multirow{2}{*}{$\begin{array}{l}\text { Data from [8] } \\
\left(\times 10^{-9} \Omega \mathrm{m}\right)\end{array}$} & \multirow{2}{*}{$\begin{array}{l}\text { Data from eqn. } 3 \\
\left(\times 10^{-9} \Omega \mathrm{m}\right)\end{array}$} & \multirow{2}{*}{ Error $(\%)$} \\
\hline (K) & $\left({ }^{\circ} \mathrm{C}\right)$ & & & \\
\hline 70 & -203 & 1.52 & 1.51 & 0.64 \\
\hline 77 & -196 & 2.00 & 2.00 & 0.02 \\
\hline 100 & -173 & 3.41 & 3.61 & 5.79 \\
\hline 110 & -163 & 4.26 & 4.31 & 1.09 \\
\hline 120 & -153 & 5.18 & 5.01 & 3.37 \\
\hline 200 & -73 & 10.5 & 10.6 & 1.31 \\
\hline 300 & 27 & 17.6 & 17.6 & 0.07 \\
\hline 400 & 127 & 24.5 & 24.6 & 0.19 \\
\hline
\end{tabular}

\subsection{Effects of temperature changes on the core} components

In a similar manner, the resistivity of the laminated steel core is dependent on the temperature. In [2], the operating resistivity of the core at temperature $T^{\circ} \mathrm{C}$ is given as:

$$
\rho_{c}=\frac{\left(1+\Delta \rho_{c} T\right) \rho_{c_{-} 20^{\circ} \mathrm{C}}}{\left(1+20 \Delta \rho_{c}\right)}
$$

where $\Delta \rho_{c}=$ thermal resistivity coefficient of the core $\rho_{c_{-} 20^{\circ} \mathrm{C}}=$ core resistivity at $20^{\circ} \mathrm{C}$.
The core resistivity at $20^{\circ} \mathrm{C}$ is $1.8 \times 10^{-7} \Omega \mathrm{m}$, and $\Delta \rho_{c}=$ $0.006 \times 10^{-8} \Omega \mathrm{m} /{ }^{\circ} \mathrm{C}$. Using eqn. 4 , at $T=-196^{\circ} \mathrm{C}$, the corresponding resistivity of the core becomes an unrealistic negative value:

$$
\rho_{c_{-}-196^{\circ} \mathrm{C}}=-2.83 \times 10^{-8} \Omega \mathrm{m}
$$

Therefore, eqn. 4 cannot be used when estimating the core resistivity at low temperatures. For the core material in the sample transformers, the exact composition of the steel material is unknown. Hence, the exact resistivity of the core material at $-196^{\circ} \mathrm{C}$ could not be determined. Instead, the core resistivity at $-196^{\circ} \mathrm{C}$ was estimated by trial and error. It was achieved by setting the resistivity of one transformer to a value such that the desired performance was obtained. It was found to be $4.5 \times 10^{-8} \Omega \mathrm{m}$. The same resistivity value was then applied to the other two transformers. Similar performances were also observed. Hence, the resistivity of the core at $-196^{\circ} \mathrm{C}$ becomes:

$$
\rho_{C_{-}-196^{\circ} \mathrm{C}}=4.5 \times 10^{-8} \Omega \mathrm{m}
$$

In addition, the skin depth of the steel core is also affected by the change in the resistivity of the core with temperature. The skin depth is defined as [9]:

$$
\delta_{e c}=\sqrt{\frac{2 \rho_{c}}{\mu_{o} \mu_{r c} \omega}}
$$

where $\mu_{o}=$ permeability of free space $=4 \pi \times 10^{-7} \mathrm{Hm}^{-1}$

$\mu_{r c}=$ relative permeability of the core

$\omega=2 \pi f$

At a power frequency of $50 \mathrm{~Hz}$ and under an ambient temperature of $20^{\circ} \mathrm{C}$, the skin depth of the laminated steel core is calculated to be $0.68 \mathrm{~mm}$. It is significantly greater than half the lamination thickness, $c_{1} / 2=0.25 \mathrm{~mm}$. Therefore, the magnetic flux can be considered to be uniformly distributed throughout the lamination and indeed the entire core cross section. This will also be true for normal operating temperatures which are higher than ambient.

However, at a temperature of $-196^{\circ} \mathrm{C}$, the calculated skin depth significantly drops to $0.23 \mathrm{~mm}$. The distribution of flux within the laminated core is no longer uniform. The flux will have a tendency to concentrate towards the outside surfaces of the laminations. Hence, immersing the transformers in liquid nitrogen causes the skin depth in the laminations of the transformer cores to decrease below half the lamination thickness, and this affects the core loss components, as detailed in the subsequent two Sections.

\subsection{Eddy current loss}

The total core loss consists of eddy current and hysteresis losses. They can each be represented by an equivalent shunt resistance. In the reverse design model, the eddy current resistance $R_{e c}$ is calculated using [10]:

$$
R_{e c}=\frac{12 \rho_{c}}{c_{l}^{2}} \frac{N_{1}^{2} A_{c}}{l_{c}}
$$

where $c_{l}=$ lamination thickness

$N_{1}=$ number of primary turns

$A_{c}=$ cross-sectional area of the core

$l_{c}=$ length of the core

Eqn. 6 assumes that the skin depth is significantly greater than half the lamination thickness. However, when the skin depth drops to below half the lamination thickness, the effective cross-sectional area of the core is reduced, the flux distribution and hence the eddy current flow can no longer be considered uniform. Therefore eqn. 6 must be modified when the skin depth is less than $c_{1} / 2$. 
To provide a more realistic calculation of the eddy current losses, consideration is given to a transformer core, with $n$ laminations, as depicted in Figs. 4 and 5. In Fig. 4, eddy current flows in each lamination, the direction of the flow being opposite to that of the current in the excitation winding due to Lenz's law. Fig. 5 shows an enlarged section of one of the laminations. When the skin depth is less than $c_{l} / 2$, the effective eddy current path length is calculated as:

$$
\begin{aligned}
l_{e c} & =2\left(w_{2}-\delta_{e c}\right)+2\left(\frac{w_{1}}{n}-\delta_{e c}\right) \\
& =2\left(w_{2}+\frac{w_{1}}{n}\right)-4 \delta_{e c}
\end{aligned}
$$

where $w_{1}, w_{2}=$ widths 1 and 2 of the core, respectively.

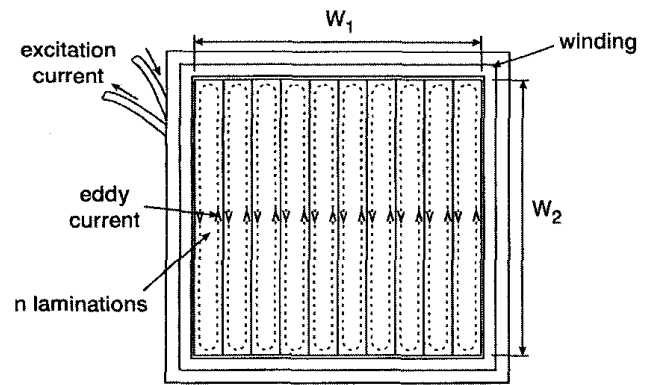

Fig. 4 Determining eddy current loss in transformer $n$ laminations

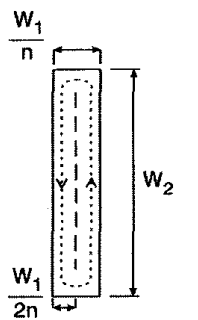

Fig.5 Determining eddy current loss in transformer

1 lamination is:

The effective eddy current resistance for one lamination

$$
R_{e c n}=\rho_{c} \frac{l_{e c}}{A_{e c n}}
$$

where $A_{e c n}=$ effective cross-sectional area of the eddy current flow path

$$
=l_{c} \times \delta_{e c}
$$

The power dissipated in the lamination is:

$$
P_{e c n}=\left(\frac{V_{c}}{n}\right)^{2} \frac{1}{R_{e c n}}
$$

where $V_{c}=$ induced voltage in the core

$$
=V_{1} / N_{1}
$$

Therefore,

$$
P_{e c n}=\frac{V_{1}^{2}}{n^{2} N_{1}^{2} \rho_{c}}\left(\frac{l_{c} \delta_{e c}}{2\left(w_{2}+\frac{w_{1}}{n}\right)-4 \delta_{e c}}\right)
$$

The total power for $n$ laminations is:

$$
\begin{aligned}
P_{e c} & =n P_{e c n} \\
& =\frac{V_{1}^{2}}{n N_{1}^{2} \rho_{c}}\left(\frac{l_{c} \delta_{e c}}{2\left(w_{2}+\frac{w_{1}}{n}\right)-4 \delta_{e c}}\right)
\end{aligned}
$$

The equivalent eddy current resistance of the core, referred to the primary, is thus:

$$
\begin{aligned}
R_{e c} & =\frac{V_{1}^{2}}{P_{e c}} \\
& =\frac{2 N_{1}^{2} \rho_{c}}{l_{c}}\left(\frac{n w_{2}+w_{1}-2 n \delta_{e c}}{\delta_{e c}}\right)
\end{aligned}
$$

\subsection{The hysteresis loss}

The hysteresis loss resistance of a partial core transformer can be calculated using [3]:

$$
R_{h}=\frac{e_{1}^{2}}{P_{h p c}}
$$

where $e_{1}=$ induced primary winding voltage

$P_{h p c}=$ partial core hysteresis power loss

$P_{h p c}$ is defined as

$$
P_{h p c}=\nu_{c}^{1+\alpha_{h p c}} \gamma_{c} k_{h p c}^{\alpha_{h p c}} f B_{c}^{x}
$$

where $v_{c}=$ volume of the core

$\gamma_{c}=$ density of the core

$\alpha_{h p c}=$ partial core factor

$k_{\text {hpc }}=$ partial core hysteresis loss constant

$x=$ Steinmetz's factor, whose value ranges between 1.8 to 2.5 [7].

The flux density $B_{c}$ is calculated as:

$$
B_{c}=\frac{\sqrt{2} V_{1}}{\omega N_{1} A_{c}}
$$

where $V_{1}=$ input voltage.

Since the effective cross-sectional area of the core is also affected by the skin depth, the area $A_{c}$ in eqn. 15 in which the flux flows is replaced by a new effective area:

$$
A_{e f f}=w_{2} w_{1}-\left(w_{2}-2 \delta_{e c}\right)\left(w_{1}-2 n \delta_{e c}\right)
$$

\section{Testing the model}

A comparison was again made between the modified calculated and experimental results. This is presented in Table 4.

The new calculated $R_{c}$ components determined from the open circuit tests are much closer to the experimental values than those of Table 2 . Therefore, the new formulations are justified. However, the calculated values of the winding resistance $R_{\text {wind }}$ are significantly different to the experimental values, and worse than the calculated values of Table 2. When performing the shortcircuit tests, the power dissipated as heat could be observed with the transformers constantly 'boiling' in the liquid nitrogen, generating gas bubbles. Therefore, even though the ambient temperature surrounding the transformers was approximately $-196^{\circ} \mathrm{C}$, the transformer windings actually operated at a relatively higher temperature. Due to the limitations of the test instruments, measuring the actual transformers' temperatures was not possible.

Using the model, the actual operating temperature of one of the transformers was predicted in order to match the experimental result. It was found to be approximately $-170^{\circ} \mathrm{C}$, an increase of $26^{\circ} \mathrm{C}$. The same temperature was then applied to the other two transformers to calculate their performance. Another comparison was made between the calculated and the experimental results. This is shown in Table 5.

The calculated results can be seen to agree reasonably well with the experimental results for all three transformers. 
Table 4: Experimental results for the transformers after resistance calculation modifications

\begin{tabular}{|c|c|c|c|c|c|c|c|c|c|}
\hline \multirow{2}{*}{$\begin{array}{l}o / c \text { and } s / c \\
\text { test results }\end{array}$} & \multicolumn{3}{|c|}{ Transformer 1} & \multicolumn{3}{|c|}{ Transformer 2} & \multicolumn{3}{|c|}{ Transformer 3} \\
\hline & Calc. & Meas. & Error $(\%)$ & Calc. & Meas. & Error $(\%)$ & Calc. & Meas. & Error (\%) \\
\hline$V_{100}, V$ & 110 & 110 & 0 & 230 & 230 & 0 & 230 & 230 & 0 \\
\hline$l_{10 c} \mathrm{~A}$ & 8.2 & 8.4 & 14 & 19.8 & 21.9 & 9 & 10.7 & 10.25 & 4 \\
\hline$P_{10 a}, W$ & 81 & 70 & 16 & 266 & 280 & 5 & 93 & 120 & 22 \\
\hline$p f_{1 o c}$ & 0.09 & 0.08 & 13 & 0.05 & 0.06 & 17 & 0.04 & 0.05 & 20 \\
\hline$R_{c^{\prime}} \Omega$ & 148.7 & 173.1 & 14 & 197.3 & 188.9 & $\cdot 4$ & 562.2 & 440.8 & 27 \\
\hline$x_{m} \Omega$ & 13.6 & 13.1 & 17 & 11.6 & 10.5 & 10 & 21.5 & 22.5 & 4 \\
\hline$V_{1 s c}, V$ & 11 & 11 & 0 & 40 & 40 & 0 & 12.2 & 12.2 & 0 \\
\hline$l_{1 s c^{\prime}} \mathrm{A}$ & 44 & 40 & 10 & 25.5 & 23 & 11 & 8.2 & 8.7 & 6 \\
\hline$P_{1 s c}, W$ & 138 & 200 & 31 & 202 & 280 & 28 & 8 & 18 & 56 \\
\hline$p f_{1 s c}$ & 0.28 & 0.44 & 36 & 0.20 & 0.30 & 33 & 0.08 & 0.18 & 56 \\
\hline$R_{\text {wind }}, \Omega$ & 0.07 & 0.13 & 46 & 0.31 & 0.53 & 41 & 0.11 & 0.23 & 52 \\
\hline$X_{\text {leak }}, \Omega$ & 0.24 & 0.24 & 0 & 1.54 & 1.66 & 7 & 1.47 & 1.38 & 6 \\
\hline
\end{tabular}

Table 5: Experimental results for the transformers: final modifications

\begin{tabular}{|c|c|c|c|c|c|c|c|c|c|}
\hline \multirow{2}{*}{$\begin{array}{l}\mathrm{o} / \mathrm{c} \text { and } \mathrm{s} / \mathrm{c} \\
\text { test results }\end{array}$} & \multicolumn{3}{|c|}{ Transformer 1} & \multicolumn{3}{|c|}{ Transformer 2} & \multicolumn{3}{|c|}{ Transformer 3} \\
\hline & Calc. & Meas. & Error $(\%)$ & Calc. & Meas. & Error $(\%)$ & Calc. & Meas. & Error $(\%)$ \\
\hline$V_{100}, V$ & 110 & 110 & 0 & 230 & 230 & 0 & 230 & 230 & 0 \\
\hline$I_{10 c} \mathrm{~A}$ & 8.2 & 8.4 & 2 & 19.8 & 21.9 & 9 & 10.7 & 10.25 & 4 \\
\hline$P_{100}, W$ & 83 & 70 & 18 & 284 & 280 & 1 & 97 & 120 . & 19 \\
\hline$p f_{1 o c}$ & 0.09 & 0.08 & 13 & 0.06 & 0.06 & 0 & 0.04 & 0.05 & 20 \\
\hline$R_{c}, \Omega$ & 145.0 & 173.1 & 16 & 186.6 & 188.9 & 1 & 546.3 & 440.8 & 23 \\
\hline$X_{m}, \Omega$ & 13.6 & 13.1 & 4 & 11.6 & 10.5 & 10 & 21.5 & 22.5 & 4 \\
\hline$V_{1 s c} V$ & 11 & 11 & 0 & 40 & 40 & 0 & 12.2 & 12.2 & 0 \\
\hline$l_{1 s c} \mathrm{~A}$ & 40 & 40 & 0 & 24.1 & 23 & 5 & 8.2 & 8.7 & 6 \\
\hline$P_{1 s c}, W$ & 216 & 200 & 8 & 342 & 280 & 20 & 15 & 18 & 17 \\
\hline$p f_{1 s c}$ & 0.49 & 0.44 & 11 & 0.35 & 0.30 & 35 & 0.15 & 0.18 & 17 \\
\hline$R_{\text {wind }}, \Omega$ & 0.14 & 0.13 & 7 & 0.59 & 0.53 & 11 & 0.22 & 0.23 & 4 \\
\hline$X_{\text {leak }}, \Omega$ & 0.24 & 0.24 & 0 & 1.55 & 1.66 & 7 & 1.47 & 1.38 & 6 \\
\hline
\end{tabular}

Table 6: Transformer 4 design data

\begin{tabular}{ll}
\hline & Transformer 4 \\
\hline Ratings & \\
$\quad$ Primary voltage, $\mathrm{V}$ & 230 \\
Secondary voltage, $\mathrm{V}$ & 25 \\
VA rating, VA & 5000 \\
$\quad$ Operating frequency, $\mathrm{Hz}$ & 50 \\
Core & \\
Length, mm & 195 \\
Width 1, mm & 39 \\
Width 2, mm & 43 \\
Lamination thickness, $\mathrm{mm}$ & 0.5 \\
Relative permeability & 2000 \\
Material density, $\mathrm{kg} / \mathrm{m} 3$ & 7870 \\
$\alpha_{\text {hpc }}$ & -2.5 \\
$k_{\text {hpc }}$ & $3.5 \times 10^{3}$ \\
$\quad x$ & 1.84 \\
Primary winding & \\
Number of layers & 9.5 \\
Wire diameter, $\mathrm{mm}$ & 1.9 \\
Secondary winding & \\
Number of layers & 3.5 \\
Wire diameter, mm & 4.0 \\
\hline
\end{tabular}

They are very similar to the values listed in Table 2 calculated using the original model, but at an operating temperature of $-196^{\circ} \mathrm{C}$. Therefore, it is justified to set the operating temperature of the transformer windings to $-170^{\circ} \mathrm{C}$.

\section{Verification of the model}

Having modified the resistance parameters in the reverse design model as described, and determined the actual operating temperature of the windings in three transformers, the effectiveness of the reverse approach under different operating temperatures can be examined. Another transformer, transformer 4, was designed and built. It was also designed for high load current applications. Its physical and electrical specifications for normal operating temperatures as well as constants of the core material are listed in Table 6.

The equivalent circuit parameters, referred to the primary, and calculated for different operating temperatures using the new derivations, are presented in Table 7. The measured values, as determined by the corresponding standard open circuit and shortcircuit tests, are also shown.

It can be seen that the results obtained from both the model and the tests agree well with each other. Transformer 4 was then operated at rated conditions to compare calculated and measured values. The results are given in Table 8 .

The closeness of the calculated and measured results for the transformer in Table 8 , both at normal and liquid 
nitrogen temperatures, further emphasises the formulations derived in the paper, and the effectiveness and flexibility of the reverse design method.

Table 7: Calculated and measured equivalent circuit parameters for the sample transformer under different operating temperatures

\begin{tabular}{|c|c|c|c|c|c|c|}
\hline \multirow{2}{*}{$\begin{array}{l}\mathrm{o} / \mathrm{c} \text { and } \mathrm{s} / \mathrm{c} \\
\text { test results }\end{array}$} & \multicolumn{3}{|c|}{ Normal temperature } & \multicolumn{3}{|c|}{ Liquid nitrogen } \\
\hline & Calc. & Meas. & Error $(\%)$ & Calc. & Meas. & Error $(\%)$ \\
\hline$V_{10 c r} V$ & 230 & 230 & 0 & 230 & 230 & 0 \\
\hline$I_{10 C^{\prime}} \mathrm{A}$ & 5.5 & 5.4 & 2 & 5.9 & 5.7 & 4 \\
\hline$P_{100}, W$ & 101 & 96 & 5 & 84 & 80 & 5 \\
\hline$p f_{1 \circ c}$ & 0.08 & 0.08 & 0 & 0.06 & 0.06 & 0 \\
\hline$R_{c} \Omega$ & 522 & 551 & 5 & 625 & 661 & 5 \\
\hline$X_{m}, \Omega$ & 42.0 & 42.6 & 1 & 39.0 & 40.3 & 3 \\
\hline$V_{1 s c}, V$ & 100 & 100 & 0 & 100 & 100 & 0 \\
\hline$I_{1 s c} \mathrm{~A}$ & 17.2 & 17.2 & 0 & 22.7 & 21.4 & 6 \\
\hline$P_{1 s c}, \mathrm{~W}$ & 1093 & 1030 & 6 & 429 & 390 & 10 \\
\hline$p f_{1 s c}$ & 0.64 & 0.60 & 7 & 0.19 & 0.18 & 6 \\
\hline$R_{\text {wind }} \Omega$ & 3.69 & 3.49 & 6 & 0.83 & 0.85 & 2 \\
\hline$X_{\text {leak }}, \Omega$ & 4.49 & 4.65 & 3 & 4.32 & 4.59 & 6 \\
\hline
\end{tabular}

Table 8: Calculated and measured rated load performance at different operating temperatures

\begin{tabular}{lllllll}
\hline $\begin{array}{l}\text { Performance } \\
\text { parameters }\end{array}$ & \multicolumn{3}{l}{ Normal temperature } & \multicolumn{3}{c}{ Liquid nitrogen } \\
& Calc. & Meas. & Error (\%) & Calc. & Meas. & Error (\%) \\
\hline$V_{1}, \mathrm{~V}$ & 234 & 234 & 0 & 222 & 222 & 0 \\
$I_{1}, \mathrm{~A}$ & 23 & 23 & 0 & 26 & 24 & 8 \\
$V_{2}, \mathrm{~V}$ & 21 & 25 & 16 & 34 & 30 & 13 \\
$l_{2}, \mathrm{~A}$. & 112 & 109 & 3 & 126 & 125 & 1 \\
$P_{1}, \mathrm{~kW}$ & 4.4 & 4.2 & 5 & 4.4 & 4.0 & 10 \\
Efficiency, \% & 56 & 60 & 7 & 87 & 92 & 5 \\
Regulation, \% & 53 & 51 & 4 & 37 & 28 & 24 \\
\hline
\end{tabular}

\section{Conclusions}

The reverse design method has been applied to partial core transformers when immersed in liquid nitrogen. Three sample transformers were designed, built and tested. From the results obtained, modifications were made to the resistance parameters of the reverse design model to account for the changes in the resistance of the materials at very low temperatures. A fourth transformer was then designed, built and tested to verify the component models. Significant agreement has been achieved between the values of the transformer equivalent circuit components as determined through calculation and test. In addition, calculated and measured operational performances of the transformer show good agreement. This paves the way for the design of partial core superconducting transformers, particularly for distribution units of various sizes and ratings.

\section{References}

1 BODGER, P.S., and LIEW, M.C.: 'Reverse as-built transformer design method', accepted for publication in Int. J. of Electr. Eng. Educ. (IJEEE), 2001

2 BODGER, P.S., LIEW, M.C., and JOHNSTONE, P.T.: 'A comparison of conventional and reverse transformer design'. Proceedings of Australasian Universities Power Engineering conference (AUPEC) Brisbane, Australia, 2000, pp. 80-85

3 LIEW, M.C., and BODGER, P.S.: 'Partial core transformer design using reverse modelling techniques', submitted to IEE Elec. Power Appl., 2001

4 FALLOU, B., BOBO, J.C., and CARVOUNAS, E.: 'Insulation components for high voltage cryoelectric equipment'. Proceedings of CIGRE international conference on Large high-voltage electrical systems, Paris, France, 1974, 25th Session, Paper 15-04, Vol. 1

5 O'NEILL, M.B., ENRIGHT, W.G., and BODGER, P.S.: 'The green-transformer; a liquid nitrogen filled power transformer'. Proceedings of EEA conference on A sustainable energy future: the engineers' challenge, Auckland. New Zealand, 2000

6 O'NEILL, M.B., ENRIGHT, W.G., and BODGER, P.S.: 'Electromechanical testing of a liquid nitrogen filled power transformer'. Proceedings of Power the new millennium conference, CEPSI 2000 Manila, Philippines, 2000

7 PAUL, C.R., NASAR, S.A., and UNNEWEHR, L.E.: 'Introduction to electrical engineering' (McGraw-Hill, Inc., Singapore, 1986)

8 DYOS, G.T. and FARRELL, T.: 'Electrical resistivity handbook: IEE materials and devices series 10' (Peter Peregrinus Ltd., London, IEE materials and devices series 10' (Peter Peregrinus Ltd., London, UK, 1992

9 SNELLING, E.C.: 'Soft ferrite: properties and applications' (Butterworth \& Co. Ltd, London, UK, 1988, 2nd edn.)

10 SLEMON, G.R.: 'Magnetoelectric devices: transducers, transformers, and machines' (John Wiley, Inc., USA, 1966) 